\title{
XXXII. A new method of investigating the resistance of the air to an oscillating sphere
}

\section{Rev. J. Challis M.A.}

To cite this article: Rev. J. Challis M.A. (1841) XXXII. A new method of investigating the resistance of the air to an oscillating sphere, Philosophical Magazine Series 3, 19:123, 229-235, DOI: $10.1080 / 14786444108650400$

To link to this article: http://dx.doi.org/10.1080/14786444108650400

册 Published online: 01 Jun 2009.

Submit your article to this journal $\sqsubset \pi$

Џ Article views: 3

Q View related articles $\asymp$ 
XXXII. A new Method of Investigating the Resistance of the Air to an Oscillating Sphere. By the Rev. J. Challis, M.A., Plumian Professor of Astronomy and Experimental Philosophy in the University of Cambridge*.

I SHOULD have been unwilling to advert again to the subject of discussion between Mr. Airy and myself, unless it had appeared to me, after reading Mr. Airy's Reply in the August Number (p.143), that something may yet be said in elucidation of this confessedly difficult question. Mr. Airy may feel assured that $I$ have no fault to find with the manner in which he supports his own views, and that equally with him I am persuaded of the necessity of adhering to strictly legitimate principles in treating questions of this nature. By placing my reasoning under another point of view, I hope to show that it is not defective in this respect.

But first I must remark, that the argument by which Mr. Airy concludes that Poisson's solution is possible and mine impossible, is nothing to the purpose. I was fully aware that my solution would not satisfy the equation by which Mr. Airy has tried it; and it was scarcely necessary to prove that an integral obtained by so experienced a mathematician as Poisson satisfied the equation from which it was derived. The question really at issue is, whether that equation, without any limitation annexed, is the one which the conditions of the problem require. The reasoning I am about to adduce will, I think, prove that it is not, and at the same time will be an answer to all that Mr. Airy has said in the latter part of his Reply.

The following general proposition will be necessary for $\mathrm{my}$ purpose :-

If $\mathrm{N}$ be a factor which makes $u d x+v d y+w d z$ an exact differential, then will

$$
\mathrm{N}(u d x+v d y+w d z)=0
$$

be the differential equation of a surface which cuts at right angles the directions of the motion of the particles through which it passes.

The proof that follows is taken from that which Mr. Earn-

is therefore somewhat too high, and it contains more than one volume of oxygen; thus it is clear that the atomic weight of carbon, as calculated from this, is also too high. That, calculated from his specific gravity, at the pressure of one-third of an atmosphere, is 75.7. He has as yet, however, only made three weighings, which he considers as little more than introductory experiments of practice. I shall be present tomorrow at the fourth determination."

* Communicated by the Author. 


\section{The Rev. J. Challis on a new Method of Investigating}

shaw has given of a very similar proposition in a memoir on Fluid Motion, contained in the Cambridge Philosophical Transactions (vol. vi. part ii. p. 204).

Let $u, v, w$ be the resolved parts in the directions of the axes of rectangular coordinates, of the velocity $\mathrm{V}$ at a point of the surface whose coordinates are $x, y, z$; and let $x+d x$, $y+d y, z+d z$ be the coordinates of another point of the surface distant by $d s$ from the former. Let the line $d s$ joining the two points make angles $\alpha, \beta, \gamma$ with the axes of coordinates, and let the direction of the velocity $\mathrm{V}$ at the point $x y z$ make the angles $\alpha^{\prime}, \beta^{\prime}, \gamma^{\prime}$ with the same axes. Then

$$
\begin{aligned}
0 & =\mathrm{N}(u d x+v d y+w d z) \\
& =\mathrm{N} V d s\left(\frac{u}{\mathrm{~V}} \cdot \frac{d x}{d s}+\frac{v}{\mathrm{~V}} \cdot \frac{d y}{d s}+\frac{w}{\mathrm{~V}} \cdot \frac{d z}{d s}\right) \\
& =\mathrm{N} \mathrm{V} d s\left(\cos \alpha \cos \alpha^{\prime}+\cos \beta \cos \beta^{\prime}+\cos \gamma \cos \gamma^{\prime}\right) .
\end{aligned}
$$

Consequently, as the factors without the brackets do not vanish, the quantity within the brackets, which is the cosine of the angle that the direction of motion makes with a tangent to the surface, must be equal to zero. Hence the motion is in the direction of a normal to the surface.

As the differential equation $\mathrm{N}(u d x+v d y+w d z)=0$, may contain the time $t$ in any manner, its integral will be of the form $\mathbf{F}(x, y, z, t)=\phi(t)$; or, differently expressed, $f(x, y, z, t)=0$. For the sake of brevity I will call the surfaces given by this equation surfaces of displacement. In any proposed instance of motion there will be an unlimited number of such surfaces, each of which will in general be continually varying its form and position, as well by the change of $t$ as by the change of form of the function $f$ depending on the arbitrary circumstances of the motion. It will be a restricted case, but sufficient for our purpose, to consider the change of a given surface of displacement to depend only on the change of $t$, the function $f$ retaining its form. Then the coordinates of the particle which at the time $t$ were $x, y, z$, will at the time $t+d t$ be $x+u d t, y+v d t, z+w d t$. Consequently, as these are coordinates of the surface of displacement in its new position,

$$
f(x+u d t, y+v d t, z+w d t, t+d t)=0 .
$$

Hence taking the letter $f$ to represent $f(x, y, z, t)$, we shall have

But

$$
f+\frac{d f}{d x} u d t+\frac{d f}{d y} v d t+\frac{d f}{d z} w d t+\frac{d f}{d t} d t=0 .
$$

$$
f=0 ; \frac{d f}{d x}=\mathrm{N} u ; \quad \frac{d f}{d y}=\mathrm{N} v ; \quad \frac{d f}{d z}=\mathrm{N} w .
$$


Hence

$$
\mathrm{N}\left(u^{2}+v^{2}+w^{2}\right)+\frac{d f}{d t}=0 ; \text { or } \mathrm{N}=-\frac{1}{\mathrm{~V}^{2}} \cdot \frac{d f}{d t} .
$$

This determination of the arbitrary factor $\mathrm{N}$ is the analytical proof that the assumed kind of motion is possible.

Let us suppose, for example, that the surface of displacement is a spherical surface, whose equation is

$$
(x-a)^{2}+(y-b)^{2}+(z-c)^{2}=\mathbf{R}^{2}
$$

$\mathrm{R}$ being its radius, and $a, b, c$ the coordinates of its centre. And first, let $a, b, c$ be constant, and $\mathrm{R}$ be a function of the time. Then

$$
\frac{d f}{d t}=-2 \mathrm{R} \cdot \frac{d \mathrm{R}}{d t}=-2 \mathrm{R} \mathrm{V} .
$$

Hence $N=\frac{2 R}{V^{-}}$, and consequently $N$ is a function of the time.

Next let $a, b$ and $\mathbf{R}$ be constant, and $c$ variable. This is to suppose that the surface of displacement is a spherical surface of given radius, the centre of which is moving parallel to the axis of $z$. For this case we have

$$
\frac{d f}{d t}=-2(z-c) \frac{d c}{d t} ; \text { and } \mathrm{V}=\frac{z-c}{\mathrm{R}} \cdot \frac{d c}{d t} .
$$

Hence

$$
\mathrm{N}=\frac{2 \mathrm{R}^{2}}{(z-c) \frac{d c}{d t}}
$$

The supposed surface of displacement being thus shown to be possible, it necessarily follows, that if a smooth solid sphere move rectilinearly in the fluid, its surface coincides with a surface of displacement. Here then we have the case of the oscillating sphere, and as $z-c$ occurs in the expression for $\mathrm{N}$, we have come to the important conclusion that $u d x$ $+v d y+w d z$ is not an exact differential in this instance, when the variation of the coordinates is from one point to another of the surface of the sphere, and therefore not an exact differential for every variation of the coordinates. It becomes so when multiplied by $\frac{R}{z-c}$, if $R$ be supposed to vary with $x, y, z$.

Let us, therefore, suppose in general that

Then

$$
\mathrm{N}(u d x+v d y+w d z)=d \phi \text {. }
$$

$$
u=\frac{1}{\mathrm{~N}} \cdot \frac{d \Phi}{d x} ; \quad v=\frac{1}{\mathrm{~N}} \cdot \frac{d \Phi}{d y} ; \quad v=\frac{1}{\mathrm{~N}} \cdot \frac{d \Phi}{d z} .
$$




\section{The Rev. J. Challis on a new Method of Investigating}

Hence by substituting these values of $u, v$, and $w$ in the equations $(a),(b),(c)$, given in page 64 of the July Number, viz.

$$
\frac{d \mathrm{P}}{d x}+k \frac{d u}{d t}=0 ; \quad \frac{d \mathrm{P}}{d y}+k \frac{d v}{d t}=0 ; \quad \frac{d \mathrm{P}}{d z}+k \frac{d w}{d t}=0 ;
$$

and neglecting quantities such as $\frac{d \mathrm{~N}}{d t} \times \frac{d \phi}{d x}$, which will be of the order of the square of the velocity, it will be found that

$$
\frac{d \mathrm{P}}{d t}+\frac{k}{\mathrm{~N}} \cdot \frac{d \Phi}{d t}=0
$$

Hence from the equation

$$
\frac{d \mathbf{P}}{d t}+\frac{d u}{d x}+\frac{d y}{d y}+\frac{d w}{d z}=0
$$

it follows (since $k a^{2}=1$ ) that

$$
\begin{aligned}
\frac{d^{2} \phi}{d t^{2}} & =a^{2} \cdot\left\{\frac{d^{2} \Phi}{d x^{2}}+\frac{d^{2} \phi}{d y^{2}}+\frac{d^{2} \Phi}{d z^{2}}\right\} \\
& -\frac{a^{2}}{\mathrm{~N}} \cdot\left\{\frac{d \mathrm{~N}}{d x} \cdot \frac{d \Phi}{d x}+\frac{d \mathrm{~N}}{d y} \cdot \frac{d \Phi}{d y}+\frac{d \mathrm{~N}}{d z} \cdot \frac{d \Phi}{d z}\right\} .
\end{aligned}
$$

Let us now transform this equation into one of polar coordinates $r, \theta, \eta$, the angle $\theta$ being that which the radius vector $r$ makes with the axis of $z$, and $\eta$ that which the plane of these two lines makes with a vertical plane. And, guided by the foregoing result, let us assume the factor $\mathrm{N}$ to be $\frac{r}{z-c}$, or $\frac{1}{\cos \theta}$. By the usual rules of transformation $I$ find that $\frac{d^{2} \cdot r \Phi}{d t^{2}}=a^{2}\left\{\frac{d^{2} \cdot r \phi}{d r^{2}}+\frac{d \cdot\left(\sin 2 \theta \frac{d \cdot r \phi}{d \theta}\right)}{r^{2} \sin 2 \theta d \theta}+\frac{d^{2} \cdot r \phi}{r^{2} \sin ^{2} \theta d \eta^{2}}\right\}$, an equation differing from Poisson's in having $\sin 2 \theta$ in the place of $\sin \theta$ in the second term of the right-hand side, and also in the signification of $\phi$. This equation, applied to the instance of the oscillating sphere, remains the same (small quantities being neglected) if the origin of the polar coordinates be the centre of the sphere. The resolved parts of the velocity in the directions of the three rectangular axes being

$$
\frac{d \phi}{d x} \cos \theta, \quad \frac{d \phi}{d y} \cos \theta, \quad \frac{d \phi}{d z} \cos \theta,
$$

the velocity in the direction of $r$ will be $\frac{d \phi}{d r} \cos \theta$. Now the 
the Resistance of the Aiv to an Oscillating Sphere. 233

conditions of the proposed problem require that when $R$ is substituted for $r$ in $\frac{d \Phi}{d r}$, the equation

$$
\frac{d \phi}{d r} \cos \theta=\frac{d c}{d t} \cos \theta
$$

should be true, whatever be $\theta$ and $\eta$. Hence it follows that $\phi$ is a function of $r$ and $t$ only, and that $\frac{d \phi}{d \theta}=0, \frac{d \phi}{d \eta}=0$. The above equation consequently becomes

$$
\frac{d^{2} \cdot r \phi}{d t^{2}}=a^{2} \cdot \frac{d^{2} \cdot r \phi}{d r^{2}}
$$

according with what precedes both in giving for $\phi$ a function of $r$ and $t$ only, and in making $\sec \theta(u d x+v d y+w d z)$ an exact differential. Hence the value of the factor $\mathrm{N}$ has been correctly assumed. Since $\frac{d \phi}{d \theta}=0$, and $\frac{d \phi}{d \eta}=0$, the velocity is wholly in the direction of $r$, and $\mathrm{V}=\frac{d \phi}{d r} \cos \theta$. But by integration, $r \phi=f(r-a t)$. Hence

$$
\mathrm{V}=\left\{\frac{f^{\prime}(r-a t)}{r}-\frac{f(r-a t)}{r^{2}}\right\} \cos \theta
$$

This is the value of $\mathrm{V}$ which $\mathrm{I}$ am contending for, and from it the result $I$ originally obtained respecting the resistance of the air to an oscillating sphere necessarily follows.

All the above reasoning, excepting the introduction of the factor $\mathrm{N}$, is according to the principles of the method adopted by Poisson. The sole reason of the difference of result is, that Poisson has argued as if $u d x+v d y+w d z$ were an exact differential in an instance, where, if my reasoning be good, that quantity is not an exact differential unless it be multiplied by a factor.

The investigation I have now gone through is in perfect agreement with the principles I advocated in my communication to the June Number of this Journal (S. 3. vol. xviii. p. 477), and may serve to place in a clear light the position $I$ have there maintained, viz. that $u d x+v d y+w d z$ cannot in general be an exact differential unless the variation of the coordinates at a given instant be in the direction of a normal to a surface of displacement. This limitation is equivalent to the introduction of the factor $\mathrm{N}$.

Being aware that $I$ am arguing for a principle, which, if true, must have a very important bearing in the treatment of hydrodynamical problems, I feel it incumbent on me to support my 


\section{On the Resistance of the Air to an Oscillating Sphere.}

views by an accumulation of evidence, and will therefore add another argument. The equations $(a),(b),(c)$, quoted above, are accurately true if the complete differential coefficients $\left(\frac{d u}{d t}\right),\left(\frac{d v}{d t}\right),\left(\frac{d w}{d t}\right)$ be substituted for the partial differential coefficients $\frac{d u}{d t}, \frac{d v}{d t}, \frac{d w}{d t}$, and become by this substitution

$\frac{d \mathrm{P}}{d x}+k\left(\frac{d u}{d t}\right)=0 ; \frac{d \mathrm{P}}{d y}+k\left(\frac{d v}{d t}\right)=0 ; \frac{d \mathrm{P}}{d z}+k\left(\frac{d w}{d t}\right)=0$. Hence at the same time that $\frac{d \mathrm{P}}{d x} d x+\frac{d \mathrm{P}}{d y} d y+\frac{d \mathrm{P}}{d z} d z$ is an exact differential, $\left(\frac{d u}{d t}\right) d x+\left(\frac{d v}{d t}\right) d y+\left(\frac{d w}{d t}\right) d z$ is an exact differential. Let therefore this latter quantity be the differential with respect to $x, y, z$, of a function $\psi$ of $x, y, z$ and $t$. Then

$$
\left(\frac{d u}{d t}\right)=\frac{d \psi}{d x} ;\left(\frac{d v}{d t}\right)=\frac{d \psi}{d y} ; \quad\left(\frac{d w}{d t}\right)=\frac{d \psi}{d z}
$$

Hence, by integration,

$$
u=\int \frac{d \psi}{d x} d t ; \quad v=\int \frac{d \psi}{d y}\left(d t ; \quad w=\int \frac{d \psi}{d z} d t .\right.
$$

Consequently, differentiating independently of the time,

$$
\frac{d u}{d y}=\int \frac{d^{2} \psi}{d y d x} d t=\int \frac{d^{2} \psi}{d x d y} d t=\frac{d v}{d x} .
$$

So $\frac{d u}{d z}=\frac{d w}{d x}$, and $\frac{d v}{d z}=\frac{d w}{d y}$.

Hence $u d x+v d y+w d z$ and $\frac{d \mathrm{P}}{d x} d x+\frac{d \mathrm{P}_{1}}{d y} d y+\frac{d \mathrm{P}}{d z} d z$ are also exact differentials at the same time. Now any one arguing according to the views maintained by $\mathrm{Mr}$. Airy would say that the latter quantity is unconditionally an exact differential; and how the conclusion could be avoided that $u d x$ $+v d y+w d z$ is without limitation an exact differential in every instance of fluid motion whatever, I am unable to see. But this conclusion is certainly untrue. Poisson has written a long memoir on the propagation of motion in elastic fluids, on the supposition that that condition is not fulfilled. (See Memoirs of the Paris Academy, tom. x. 1831.) The explanation of this apparent contradiction I believe to be as follows:When the complete differential $(d \mathrm{P})$ is substituted for the sum 
of the partial differentials $\frac{d \mathrm{P}}{d x} d x, \frac{d \mathrm{P}}{d y} d y, \frac{d \mathrm{P}}{d z} d z$, it is tacitly assumed that $\mathrm{P}$ is a function of $x, y, z$, which varies continuously, and not arbitrarily, with the variation of the coordinates, and so far as the variation of the function is such, the substitution is legitimate. Now in any instance of fluid motion it is only in the direction of the displacement of the fluid that the variation of the velocity and density at a given time is in any respect necessarily continuous and not altogether dependent on the arbitrary displacement. In this direction it depends in part on the propagation of the motion, and on the mutual inclination to each other of the normals to the surface of displacement, in a manner which it is the peculiar province of the integration of a partial differential equation to make known, and so as to leave the particular form of the function expressing the arbitrary displacement indeterminate. But the variation of velocity and density at a given instant from one point to another along a surface of displacement altogether depends on the arbitrary disturbance, and cannot therefore be given by a similar process of integration. With the abovementioned limitation respecting the direction of variation of the coordinates, both $\frac{d \mathrm{P}}{d x} d x+\frac{d \mathrm{P}}{d y} d y+\frac{d \mathrm{P}}{d y} d z$, and $u d x$ $+v d y+w d z$ are exact differentials in every instance of fluid motion, excepting where the motion is not essentially different from that of a solid.

After this communication I shall not again enter upon the consideration of the problem which has given rise (not, I hope, without some advantage to the cause of science) to so prolonged a discussion. But the general principle on which my solution rests is too important to be hastily dismissed, and I shall probably take other opportunities of exemplifying it.

Cambridge Observatory, August 19, 1841.

XXXIII. Note on the Production of Sulphuretted Hydrogen bythe Action of decomposing Animal Matter upon Sulphates. By Robert Mallet, Ph. D., M.R.I.A.

Sir,

To Richard Phillips, Esq., \&. \&c.

THE valuable communication in the Number of the Philosophical Magazine for this month (July) by Mr. Daniell, on the spontaneous evolution of sulphuretted hydrogen in the waters of the African Coast, \&c., has ibeen read by me with peculiar interest, from its connexion with a subject to which 